\title{
Oscillatory switching under a rapidly varying magnetic field
}

\author{
Ching-Ray Chang \\ Department of Physics, National Taiwan University, Taipei, Taiwan, Republic of China \\ Jyh-Shinn Yang \\ Division of General Education, National Taiwan Ocean University, Keelung, Taiwan, Republic of China
}

(Received 4 March 1996)

\begin{abstract}
The dynamic response of magnetization under a rapidly varying applied field was analyzed using the Landau-Lifshitz equation. The computed angular dependence of the lower bound of the dynamic switching field is found to be less than the static limit and, moreover, it is asymmetric with respect to $45^{\circ}$ deflection of the applied field. Oscillatory switching occurs between the dynamic and static thresholds. The width of the reversal bands depends on the damping rate and rise time of the field. [S0163-1829(96)05641-X]
\end{abstract}

The problem of magnetization reversal in single-domain particles (SDP's) has been extensively studied for decades. ${ }^{1-5}$ This lasting interest originates partially from fundamental research and partially from information storage applications, in particular, due to the fact that the size of current high-density recording media falls within the range of SDP's and, indeed, already approaches the quantum limit. ${ }^{6}$ The damping effect on SPD switching is thus of importance to further increase the recording density in rigid disk drives and to the improvement of high-frequency performance. ${ }^{7}$ Moreover, recent experiments ${ }^{8}$ suggest that switching over a single energy barrier is not an adequate description of the observed results and detailed theoretical analysis of the dynamical switching process in SDP's is thus of considerable significance.

Two methods are commonly adopted in studies of the spin orientation in single-domain particles. One method applies a variational principle to Brown's equation and the reversal modes, and their corresponding nucleation fields are then identified from the linearized Brown's equation as an eigenvalue-eigenmode problem. ${ }^{3}$ An alternative approach is to solve the phenomenological Landau-Lifshitz-Gilbert dynamic equation of motion with suitable damping terms ${ }^{4}$ chosen in such a way that the magnetization magnitude is conserved. It was believed that for an overdamped system two methods give similar results while for an underdamped system, even in the case of an isolated particle, the dynamic reversal process has not been, because of its complexity, studied in detail. For a slowly varying applied field, the static and overdamped limits yield approximately the same results. However, the state-of-the-art high-density recording places the recording medium into high-speed switching environments and the system then usually does not have enough time to relax to its equilibrium position. ${ }^{5}$ A previous dynamic analysis with a finite rise time and small damping rate indicated that the static analysis usually overestimates the switching field. ${ }^{5,7}$ The present paper concentrates on the seldom discussed oscillatory switching occurring between the dynamic and static thresholds. It will be shown later that the width of the reversal band critically depends on the damping rate and on the rise time of the steplike field.
The dynamical switching properties are studied here using a model of uniform magnetization rotation with uniaxial anisotropy. ${ }^{1}$ We consider a single-domain uniaxial particle whose magnetization $\vec{M}$ reverses by coherent rotation. The particle has energy $E=K V \sin ^{2} \theta-V \vec{M} \cdot \vec{H}_{a}$, where $V$ is the volume and $K$ the anisotropy constant. The applied field $\vec{H}_{a}$ deviates from the easy axis by an angle $\beta$, and $\theta$ is the angle spanned by the particle's easy axis and its magnetization vector $\vec{M},|\vec{M}|=M_{s}$ by assumption, where $M_{s}$ is the saturation magnetization. The effective magnetic field $\vec{H}_{\text {eff }}$ is written as

$$
\vec{H}_{\mathrm{eff}}=-\frac{1}{V} \frac{\delta E}{\delta \vec{M}(\vec{r})} .
$$

In equilibrium the magnetization is parallel to the effective field $\vec{H}_{\text {eff }}$ and the net torque $\vec{M}(\vec{r}) \times \vec{H}_{\text {eff }}(\vec{r})$ vanishes. If the applied field either rotates or varies its magnitude, then the magnetization vector will follow a dynamic evolution to a new equilibrium. The dynamic equation usually employed is the phenomenological Landau-Lifshitz dynamic equation

$$
\frac{d \vec{M}(\vec{r})}{d t}=-\gamma\left[\vec{M}(\vec{r}) \times \vec{H}_{\mathrm{eff}}+\frac{\alpha}{\left|M_{s}\right|} \vec{M}(\vec{r}) \times\left[\vec{M}(\vec{r}) \times \vec{H}_{\mathrm{eff}}\right]\right],
$$

where $\gamma$ is the gyromagnetic ratio and $\alpha$ is a damping constant. The first term corresponds to the gyromagnetic electron spin motion and the second term represents the phenomenological contribution of the dissipative forces; its form ensures conservation of $|\vec{M}(\vec{r})|$. Commonly used ${ }^{4}$ is the mathematically equivalent Gilbert's equation.

For a large applied field there exists only one energy minimum. At moderate fields two equilibrium orientations exist and the magnetization stays at the equilibrium which is inherited from memory (hysteresis). It was believed that at zero temperature the magnetization cannot cross the energy barrier to the alternate minimum and that it should stay along its original orientation. ${ }^{4}$ For an isolated particle it was also believed that neither the rate of decrease of the field nor the damping rate $\alpha$ affects final equilibrium state. ${ }^{4}$ In an over- 
damped system or in a slowly varying applied field the magnetization is always in "quasiequilibrium" with external forces and the relaxation behavior of magnetization is therefore correctly described by the overdamped limit, e.g., Ref. 4. In high-speed switching fields, on the other hand, or in an underdamped system, the final equilibrium state is found to depend on the dissipation rate and on the variation of the energy configurations and recent studies of high-speed switching show some interesting results which cannot be explained from the static models. ${ }^{5,8}$

We model a high-speed switching field by a stepwise function; at constant angle $\beta$ the applied field suddenly switches from zero to $-H_{0}$. In reality, of course, the rise time $\tau$ is finite but extremely short, e.g., on the order of $10 \mathrm{~ns}$ for a head current rise time. ${ }^{7}$ Because the field and energy configuration change so abruptly, the magnetization has no time to respond and remains trapped in the old equilibrium state which can, therefore, be taken as an initial condition $\theta_{i}$ in the new energy configuration under $-H_{0}$; at large angles $\beta$ the initial energy corresponding to $\theta_{i}$ is usually higher than that of the barrier at $\theta_{b}$. The magnetization vector precesses along the $\vec{H}_{\text {eff }}$ direction while energy is gradually dissipated. If the dissipation process is fast, then the magnetization will always be trapped at the nearest local minimum, as predicted by the the static theory. However, if the system does not lose too much energy before $\vec{M}$ approaches $\theta_{b}$, then the magnetization may overcome the barrier and reach the other local minimum. In the case of very slow dissipation the magnetization may even traverse the barrier again and settle at the original local minimum or, indeed, in the extreme case many barrier passages may take place before the magnetization is trapped at one of the local minimum. Our observation time is usually much longer than the barrier traversal time and we only observe the final magnetization orientation: An odd number of barrier traversals is observed as a magnetization reversal while for an even number the observer claims that magnetization did not switch.

Numerical solutions of Eq. (2), expressed in Cartesian coordinates so as to avoid numerical errors at $\theta \approx 0$, were found using the fourth-order Runge-Kutta method in double precision; conservation of the magnitude of magnetization was checked after each iteration. In these calculations the magnetization was initially oriented along the easy axis direction at zero field. The field strength was linearly changed to $-H_{0}$ within the rise time $\tau$ and the evolution of magnetization was then traced until $\vec{M}$ was found trapped at a local minimum; in all cases the iteration step was gradually re-
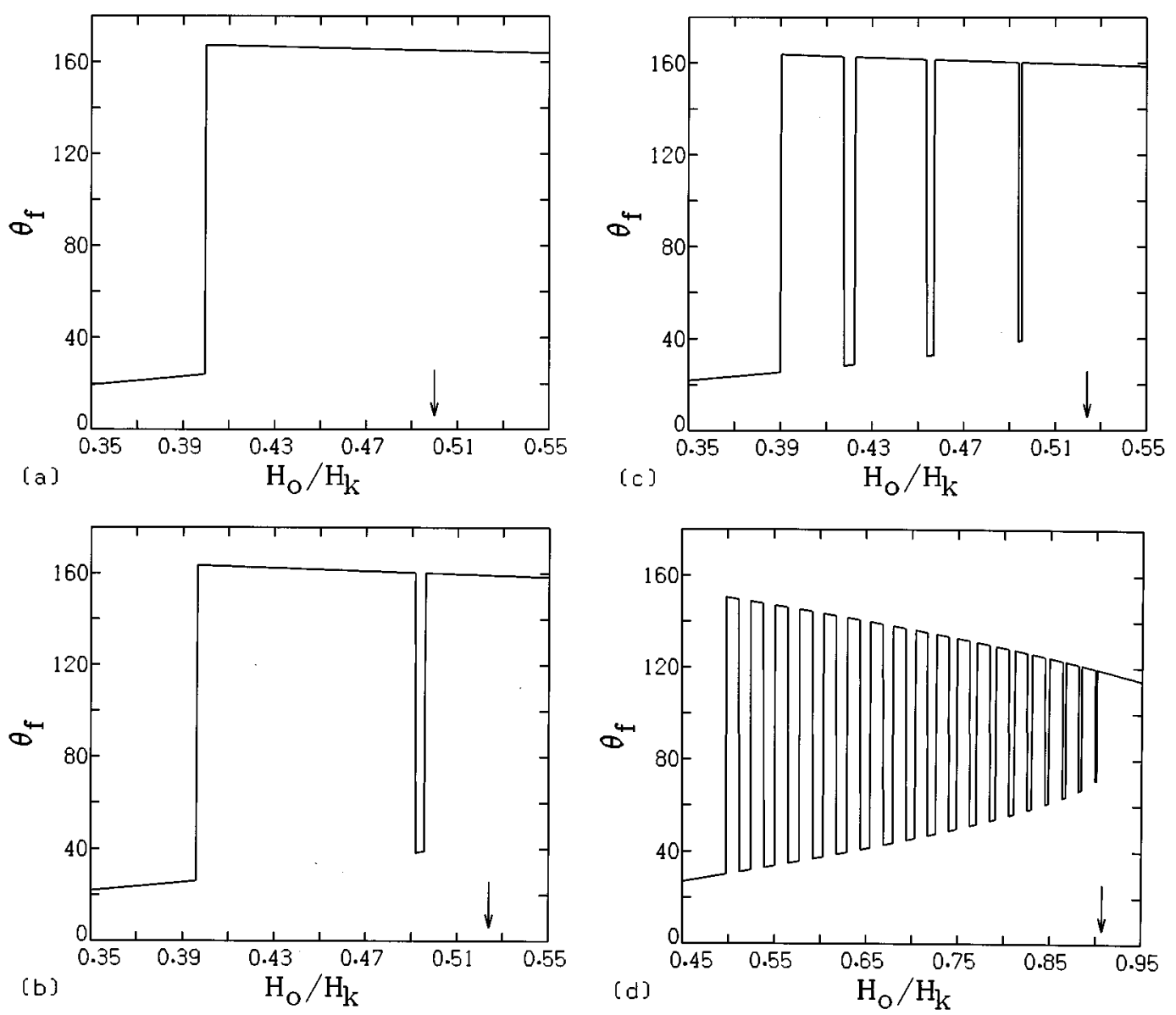

FIG. 1. Final stationary orientation (solid lines) of magnetization vs the strength of the stepwise field $H_{0}$. The arrow marks the Stoner-Wohlfarth limit for the switching field $H_{k}=2 K / M_{s}$ and rise time $\tau=0.1 / \gamma H_{k}$ in all cases. (a) $\beta=45^{\circ}$ and $\alpha=0.03$. Magnetization switches at a well-defined critical field. (b) $\beta=60^{\circ}$ and $\alpha=0.03$. Some intervals of $H_{0}$ can switch the magnetization at a field lower than the Stoner-Wohlfarth limit. (c) $\beta=60^{\circ}$ and $\alpha=0.01$. The range of the switching field becomes oscillatory. (d) $\beta=89^{\circ}$ and $\alpha=0.01$. The oscillatory range of the switching field becomes more significant and uniform. 

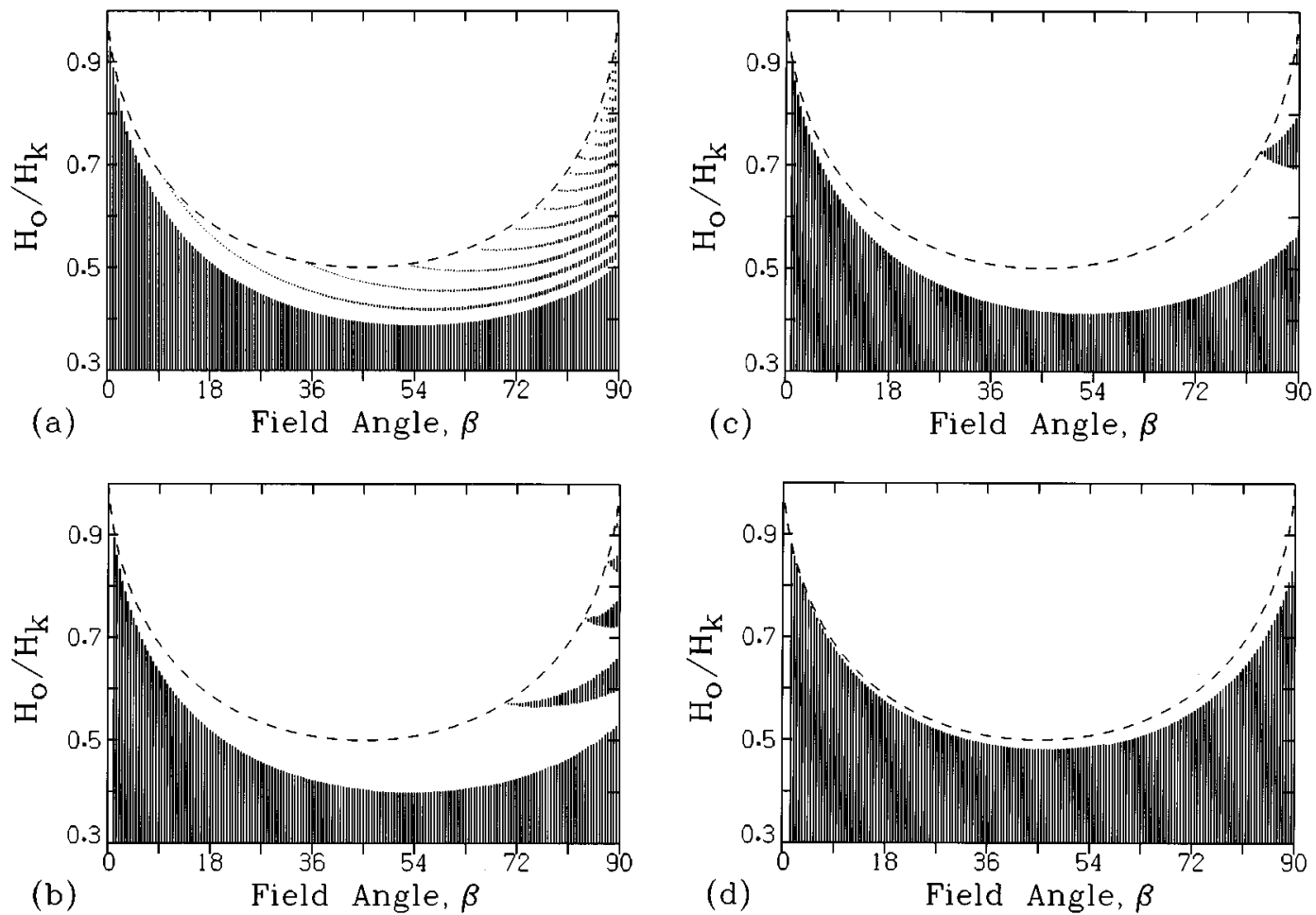

FIG. 2. Angular dependence of the switching field for stepwise applied field. Initial angle $\theta_{i}$ is along the easy axis and unshaded areas are the reversal regions. Dashed line is the Stoner-Wohlfarth limit. The rise time $\tau=0.1 / \gamma H_{k}$ and damping constants $\alpha$ are 0.01 (a), 0.05 (b), 0.1 (c), and 0.5 (d), respectively.

duced until the final result saturated. The calculation time depends on the used parameters; however, it can be done at the advanced PC within the reasonable waiting time.

In Fig. 1 we present the relationship between $H_{0}$ and the orientation of the magnetization at equilibrium, $\theta_{f}$. For a large damping constant and small $\beta$ the energy dissipates fast and the magnetization can only switch at a critical field [Fig. 1(a)]. When damping becomes weaker, however, then the magnetization switches within several intervals of $H_{0}$ [Fig. 1(b)]. A lower damping rate shifts the lower bound of the switching field to smaller values. The oscillatory switching behavior of the magnetization with $H_{0}$ becomes more significant, at large $\beta$ [Fig. 1(c)]. According to Fig. 1(d), further, the interval of $H_{0}$ becomes very short at large $\beta$ and small damping. Obviously, if we define the switching field as the lower bound of $H_{0}$ in Fig. 1, then the switching field depends on both the energy loss rate and on the angle of applied field. Angular dependence of the applied field strength $H_{0}$ on applied field angle is shown in Fig. 2 for reversal (unshaded) bands with different damping constants. The present dynamic analysis shows that until $H_{0}$ reaches the Stoner-Wohlfarth limit the final orientation of magnetization alternates. The lower bound of the reversal band is asymmetric with respect to the angle $\beta=45^{\circ}$ and large deviations from the Stoner-Wohlfarth limit are observed at large $\beta$. On increase of damping rate the dynamic lower bound gradually approaches the static limit and the reversal band gap increases. The width of band gap thus reflects the amount of energy dissipated during the reversal processes. We have also analyzed the influence of distribution of easy axis orientations and for a noninteracting assembly we found that the oscillatory switching behavior disappears at large dispersions of the easy axes.
Due to the strong nonlinearity of the equations of motion, the final orientation of the magnetization is very sensitive to the initial state (see Fig. 3) and $\theta_{i}$ can be fluctuating due to either interparticle interactions or to thermal agation. The sensitivity to even small perturbations of the initial state indicates that thermal fluctuations are apparently very important for the dynamical evolution of magnetization. In the

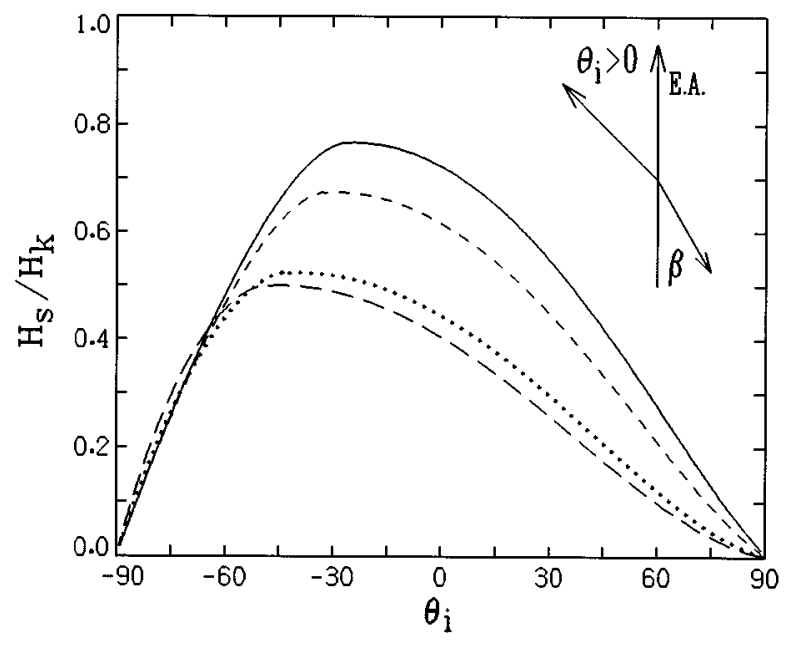

FIG. 3. Angular dependence of the dynamical threshold of the switching field $H_{s}$ for selected initial deviations from the easy axis. Positive value is away from the applied field direction (i.e., azimuthal angle $\phi=0)$ and negative value is close to the applied field direction $(\phi=\pi)$. Parameters used here are $\beta=5^{\circ}$ (solid line), $10^{\circ}$ (short-dashed line), $30^{\circ}$ (dotted line), and $45^{\circ}$ (long-dashed line), and $\alpha=0.05$ and $\tau=0.1 / \gamma H_{k}$. 
presented calculations we set, for simplicity, initial conditions with zero azimuthal angle. Its variation produces the similar but more complicated results as the variation of $\theta_{i}$ shown in Fig. 3. We have also calculated the influence of the rise time $\tau$ on the structure of the reversal band and found it to be of lesser influence on the oscillatory switching property than the damping constant $\alpha$. A large $\tau$ increases the dynamical threshold and decreases the number of the reversal bands.

In summary, we have analyzed the dynamical reversal process of magnetization under high-speed switching fields and we have shown that the static analysis is valid only in the overdamped limit while the switching behavior under a high-speed field can be very complicated indeed. The angular dependence of the lower bound of the dynamic switching field is found to be not only asymmetric but also lower than the Stoner-Wohlfarth limit and in the field interval between the dynamical and static lower bounds the switching behavior of magnetization is found to critically depend on the detailed dynamics of the system. If the energy of the initial state is high enough and damping rate is low, then oscillatory phenomena become significant. On the simple example of coherent rotation of magnetization we have demonstrated the complexity of the switching properties which can experimentally be verified ${ }^{5,8}$ only by full range explorations of the field strength $H_{0}$. In the case of nonuniform rotation the topological dimension of the global attractor for the Landau-Lifshitz equations is large ${ }^{9}$ and dynamical analysis becomes very difficult even at zero temperature. ${ }^{10} \mathrm{~A}$ clear interpretation of experimental data therefore requires a full dynamical analysis based on sufficient information about the system.

We have also demonstrated the sensitivity of reversal properties on the initial magnetization orientation and we have shown that even small deviations can significantly affect the whole reversal behavior, especially under the highspeed switching field. This suggests that at finite temperatures, where thermal agitation is of significance, even a full dynamical analysis may possibly lead to spurious results under high-speed switching fields. For high-density recording media, therefore, a full dynamical analysis with thermal agitations included is required.

This research has been partially supported by NSC Grant No. NSC-85-2112-M002-03, ROC.
${ }^{1}$ E. C. Stoner and E. P. Wohlfarth, Philos. Trans. R. Soc. London, Ser. A 240, 599 (1948).

${ }^{2}$ W. F. Brown, Phys. Rev. 130, 1677 (1963)

${ }^{3}$ A. Aharoni, Rev. Mod. Phys. 34, 227 (1962).

${ }^{4}$ H.N. Bertram and J.-G. Zhu, in Solid State Physics, edited by H. Ehrenreich and D. Turnbull (Academic, New York, 1992), Vol. 46, pp. 271-371.

${ }^{5}$ W. D. Doyle, P. J. Flanders, and L. He, IEEE Trans. Magn. MAG-29, 3634 (1993); L. He, W. D. Doyle, and H. Fujiwara, ibid. MAG-30, 4086 (1994).
${ }^{6}$ S. Y. Chou, P. R. Krauss, and C. Kong, J. Appl. Phys. 79, 6101 (1996).

${ }^{7}$ Y. Nakatani, N. Hayashi, Y. Uesaka, and H. Fujiwara, Jpn. J. Appl. Phys. 33, 6546 (1994).

${ }^{8}$ M. Lederman, S. Schultz, and M. Ozaki, Phys. Rev. Lett. 73, 1986 (1994); M. Lederman, D. Fredkin, R. O’Barr, M. Ozaki, and S. Schultz, J. Appl. Phys. 75, 6217 (1994).

${ }^{9}$ T. L. Gill and W. W. Zachary, Phys. Lett. A 128, 419 (1988).

${ }^{10}$ B. Yang and D. R. Fredkin, J. Appl. Phys. 79, 5755 (1996). 